\title{
$\angle$ Research Square \\ Effects of Interlayer Spacing and Oxidation Degree of Graphene Oxide Nanosheets on Water Permeation: A Molecular Dynamics Study
}

qiong tan

Zhejiang $A$ and $F$ University

Yan Fan

Zhejiang $A$ and $F$ University

Zailing Song

Zhejiang $A$ and $F$ University

Junlang Chen ( $\square$ chenjunlang7955@sina.com )

Zhejiang A \& F University https://orcid.org/0000-0001-9238-781X

Liang Chen

Zhejiang $A$ and $F$ University

Research Article

Keywords: GO nanosheets, Nanofiltration, Water flow, Interlayer spacing, Oxidation degree

Posted Date: June 25th, 2021

DOl: https://doi.org/10.21203/rs.3.rs-595688/v1

License: (c) (i) This work is licensed under a Creative Commons Attribution 4.0 International License.

Read Full License

Version of Record: A version of this preprint was published at Journal of Molecular Modeling on February 8th, 2022. See the published version at https://doi.org/10.1007/s00894-022-05045-7. 


\section{Abstract}

Graphene oxide (GO) membranes have shown great potential in the applications of water filtration and desalination. The flow behavior and structural properties of water molecules through $\mathrm{GO}$ nanochannels are still under debate. In this work, molecular dynamics simulations were performed to explore the effects of interlayer spacing and oxidation degree of GO nanochannels on water transport. The results show that GO nanosheets has strong adsorption capacity. The adsorbed layer of water molecules on GO surface is thermodynamically stable and not easy to flow. When the interlayer spacing falls into the range of 0.6 1.0 nm, water molecules form into single or double adsorbed layers between two GO nanosheets. When the interlayer spacing is bigger than $1.2 \mathrm{~nm}$, the other water layers in the middle of nanochannel become disordered. Taking the separation performance based on size exclusion into consideration, the most suitable interlayer spacing for water nanofiltration is $1.2 \mathrm{~nm}$, which has one flowing layer of water molecules. Oxygen-containing groups are unfavorable for water permeation, as more and more hydrogen bonds prevent water flowing on GO surface with the increasing oxidation degree. Our simulation results may help to improve the design of GO nanofiltration membranes for water treatment.

\section{Introduction}

Graphene has unique optical, electronic and mechanical properties as well as planar structure, and can be used for nano-filtration, water treatment, supercapacitors and photocatalysis[1-5]. Graphene layered membranes are formed by stacking single layers of graphene with nanoscale interlayer spacing[6, 7]. This special laminated structure allows the permeance of water molecules and reject other molecules. The performance of desalination has been extensively studied by experimental and theoretical calculations $[8,9]$. Han et al. experimentally designed negatively charged ultrathin graphene nanofiltration membranes for water purification and found that pure water had high permeation rates and high rejection up to $99 \%$ for organic dyes and moderate rejection of $20 \% \sim 60 \%$ for salt ions [10]. In addition, since graphene is difficult to be exfoliated and easily agglomerated, its performance can be adjusted by introducing functional groups for efficient desalination, among which graphene oxide (GO) has been widely studied.

$\mathrm{GO}$ is the oxidation of pristine graphene (PG) with similar two-dimensional structure, which can be prepared by chemical and electrochemical oxidation methods $[11,12]$. There are various oxygencontaining groups on the surface of graphene oxide, such as hydroxyl, epoxy and carboxyl groups, and different oxidation processes will affect the distribution of oxygen functional groups on GO's surface and the degree of oxidation[13, 14]. GO membranes have obtained much attention due to its simple fabrication process, low price and adjustable interlayer spacing. Under dry conditions, the interlayer spacing of GO membranes is $\sim 0.8 \mathrm{~nm}$, and the slit width can be increased to $\sim 1.3 \mathrm{~nm}$ in humid air or when immersed in water due to hydration[15]. Nair et al. found that water penetration through GO membranes was not hindered, while helium could not pass GO membranes[16]. Joshi et al. reported that small ions could pass the GO membrane rapidly[17]. Recent studies[18-20] showed that GO membranes 
were modified with different functional groups or intercalated by small molecules could adjust their interlayer spacing to achieve the separation of specific organic molecules and ions in wastewater[21].

In order to further develop the above PG/GO membranes for practical processes, a fundamental understanding of the structures and properties of water or other molecules in PG/GO layered nanochannels is necessary. Since the molecular behavior in the confined space at such a nanoscale is not easily observed by existing experimental methods, thus molecular dynamics (MD) simulation has been an effective method to study the confined space at nanoscale[22-28]. It was previously reported[29, 30] that MD was used to demonstrate the flow behavior of water molecules through membranes based on graphene nanochannels, and it was found that water molecules could undergo rapid transport, mainly due to the very weak interaction between graphene surface and water molecules. The study of water permeation in GO nanochannels showed that the large number of oxygen functional groups in the nanocapillary facilitated the formation of hydrogen bonds by water molecules, leading to the enhanced water adsorption and increased water flux in the membrane pores[31, 32]. On the other hand, Yang et al. [33] found that the water flow rate in the GO membranes was low and the water molecules in the nanochannels became more disordered with the increase of oxidation, while the structure and kinetic behavior of water molecules changed significantly in $0.6-1.5 \mathrm{~nm}$ ultra-microporous pores [34]. The study of their microscopic behavior in GO membranes is still a frontier in scientific society.

In this work, we explored the structural and kinetic behavior of water molecules in PG/GO nanochannels with varied interlayer spacing of $0.6-1.8 \mathrm{~nm}$ and oxidation degree of $0 \% \sim 40 \%$, using MD simulations. The purpose is to find suitable interlayer spacing and oxidation degree for water permeation. The simulation results may be useful to improve the design of PG/GO nanochannels for the applications of water purification and desalination.

\section{Materials And Methods}

As shown in Fig. 1(A), to simplify GO model, we used epoxy and hydroxyl groups randomly distributed on GO surface to represent the oxidation of PG. The size of PG was $2.2 \times 3.2 \mathrm{~nm} 2$. Following this model, the oxidation degree (OD) of GO was defined as $\mathrm{OD}=\mathrm{NO} / \mathrm{NC} \times 100 \%$, where $\mathrm{NO}$ and $\mathrm{NC}$ are the numbers of oxygen and carbon atoms. We constructed five kinds of GO nanosheets with varied OD, namely, $0 \%(P G)$, 10\% (labeled as G010), 20\% (labeled as G020), 30\% (labeled as G030) and 40\% (GO40).

The configuration of the simulation system was presented in Fig. 1(B). Two GO nanosheets placed parallelly with each other were employed as $\mathrm{GO}$ membrane. The interval between two sheets was used as nanochannel to transport water molecules. The interlayer spacing was set at $0.6,0.8,1.0,1.2,1.4,1.6$ and $1.8 \mathrm{~nm}$, respectively. Initially, the feed side (see Fig. 1(A), Box1) was filled with pure water, while the right side (Box2) was empty. A plate was introduced into the left end of Box1 to block water from flowing between Box 1 and Box2. During the water permeation process, the plate was pushed at a speed of 0.35 $\mathrm{nm} \cdot \mathrm{ns}-1$ from left to right. 
All MD simulations were performed under the canonical ensemble (NVT) using GROMACS software (version 5.1.4)[35, 36] and OPLS-AA force field[37]. The parameters of PG were developed by Safaei et al[38]. Water was depicted by the simple point charge model[39]. During the simulation, PG and GO were position-restrained by a spring of $1000 \mathrm{kJmol}^{-1} \mathrm{~nm}^{-2}$. Periodic boundary conditions were applied in $y$ direction. The long-range electrostatic interactions were calculated by Particle-Mesh Ewald method[40, 41], and the van der Waals ( $\mathrm{vdW}$ ) interaction was computed with a cut-off of $1 \mathrm{~nm}$. The bond lengths in PG/GO and water molecules were constrained by methods of LINCS[42] and SETTLE[43], respectively. A time step of $1 \mathrm{fs}$ was used to acquire higher accuracy. The production run of each system lasted $20 \mathrm{~ns}$ to collect data for subsequent analysis.

\section{Results And Discussion}

\subsection{Adsorption of water molecules on PG/GO surface}

Prior to study the water transport through GO membranes, we should understand the adsorption features of water molecules on PG/GO surface. We therefore first performed simulations of water with single PG or $\mathrm{GO}$ nanosheets. Taking PG as an example, there are two peaks in the molecular number density profile, as shown in Figure 2(A). The first peak is localized at $z=0.33 \mathrm{~nm}$, close to the $\mathrm{vdW}$ radii of carbon and oxygen atoms. The corresponding number density is $83.5 \mathrm{~nm}-3$, substantially bigger than that of bulk water (33.4 nm-3), confirming the strong adsorption ability of PG. The second peak exhibits much weaker, about $42.4 \mathrm{~nm}-3$ at $z=0.63 \mathrm{~nm}$. The two peaks indicate that there are two adsorbed layers of water on PG surface. To understand the adsorption stability of water for PG, the mean sojourn time (MST) of water molecules confined to their initial points in z-direction was analyzed, which is defined as

$$
\operatorname{MST}(z)=t \mid \Delta z(t)<\Delta_{\mathrm{c}}
$$

where $\Delta z(t)=\left|z\left(t+t_{0}\right)-z\left(t_{0}\right)\right|$ and $\Delta_{\mathrm{c}}$ is set at $0.01 \mathrm{~nm}$. MST is a good physical quantity for characterizing the adsorption intensity. MST profiles hold the same trend with those of number density (Figure 2(B)). The maximal MST of PG is about $0.022 \mathrm{~ns}$ at $0.33 \mathrm{~nm}$, which is well consistent with the molecular number density profile. This coincidence indicates that water molecules are thermodynamically stable in the first adsorbed layer. However, the second peaks in two profiles show much weaker, just sightly higher than those in bulk solution, implying that PG's adsorption intensity weakens quickly with the increasing distance from its surface. Water on GO surface exhibits similar results, which illustrate that PG and GO possess strong adsorption capacities close to their surface $(\mathrm{d}<0.5 \mathrm{~nm})$.

\subsection{Distribution of water in nanochannels}

Then, we take two sets of systems as examples to investigate the effects of interlayer spacing and oxidation degree on water permeation through GO membranes, namely, the fixed oxidation degree $(O D=20 \%, G 020$, Figure $3 \mathrm{~A})$ with different interlayer spacing $(0.6 \sim 1.8 \mathrm{~nm})$ and the same interlayer spacing 
$(d=1.2 \mathrm{~nm}$, Figure $3 \mathrm{~B})$ with different oxidation degree (0 40\%). Figure $3 \mathrm{C}$ and $3 \mathrm{D}$ show the number density of water molecules in the nanochannels along the thickness direction (z-axis). When the interlayer spacing is only $0.6 \mathrm{~nm}$, there is just one layer of water molecules in the nanochannel. Moreover, the molecular number density is highly $200 \mathrm{~nm} 3$. Except $d=0.6 \mathrm{~nm}$, the peaks of the density profiles are close with each other and symmetrical about bilayer center at a fixed OD, falling in the range of $88 \sim 108 \mathrm{~nm} 3$, which are slightly bigger than that on single PG/GO surface. This is because the pressure in the nanochannels is much greater than 1 bar, when water molecules are pushed by the plate at a constant speed. The symmetrical two peaks correspond to the adsorption layers clinging to the $\mathrm{GO}$ surface. When $\mathrm{d}=0.8$ and $1.0 \mathrm{~nm}$, water molecules form into two layers in the nanochannel. The structure of these single or double layers of water sandwiched by PG/GO is ice-like. Hence, these layers of water are not easy to flow on PG/GO surface. When $d=1.2 \mathrm{~nm}$, water molecules form into three layers. However, when $\mathrm{d}$ is greater than $1.4 \mathrm{~nm}$, the water structures become gradually disordered in the middle of the interlayer gallery (Figure 3C). At a fixed interlayer spacing of $1.2 \mathrm{~nm}$, the density profiles are coincident that there are three layers of water between two G020 sheets. The main difference is the peak values drop slightly with the increasing OD (see Figure 3D) because of steric effects, as more and more oxygen-containing groups are evenly distributed on graphene surface. On the other hand, the middle peaks are much lower, since the adsorption intensity weakens with the increasing distance to $\mathrm{GO}$ surface.

\subsection{Water flow}

We then drive water flow by pulling a plate from left to right. Figure 4 shows the number of water molecules in Box2 increases with the simulation time. We start counting when water molecules reach Box2, and only count for the following 5ns. Interestingly, it is found that the curves are parallel with each other in all systems, regardless of channel width and oxidation degree, indicating that the curves have the same slope (i. e., water flow). Since we pull an identical plate with the same speed (see Methods), all systems exhibit similar water flow of $306 \pm 7 \mathrm{~ns}-1$. The only exception is $d=0.6 \mathrm{~nm}$, as the channel is so narrow that an extremely strong pull force is required to exert on the plate, resulting in the deformation of the plate and the water flowing across the channel becomes irregular.

\subsection{Distribution of water in nanochannels}

Although we pull the plate at the same speed, the pull forces vary with different interlayer spacing and oxidation degree, as shown in Figure 5. In general, with the fixed OD, the cumulative average pull forces are getting weaker with the increasing interlayer spacing. The pull forces continue to weaken from $6.5 \times 105 \mathrm{~kJ} / \mathrm{mol} / \mathrm{nm}$ to $3.0 \times 103 \mathrm{~kJ} / \mathrm{mol} / \mathrm{nm}$, corresponding to the channel widening from $0.6 \mathrm{~nm}$ to 1.8 $\mathrm{nm}$ (see Figure $5 \mathrm{~A}$ ). This is simply because the wider the channels are, more easily water molecules can pass. It should be noted that the interlayer spacing of GO membrane with one layer water molecules is larger than $0.8 \mathrm{~nm}$, which is confirmed by XRD experiments. Therefore, water cannot spontaneously permeate into the nanochannel, when $\mathrm{d}=0.6 \mathrm{~nm}$. Thus, only exerting a huge pressure that can water pass through such narrow channel. Furthermore, at a fixed channel width, the pull forces become stronger with 
the increasing OD. In Figure 4B, the pull forces strengthen continually from $2.2 \times 103 \mathrm{~kJ} / \mathrm{mol} / \mathrm{nm}$ to $1.3 \times 104 \mathrm{~kJ} / \mathrm{mol} / \mathrm{nm}$ with the OD rising from 0 to $40 \%$.

It has been reported that water transport through interlayer gallery is inhibited by a dominant side-pinning effect originated from the oxidized regions of $\mathrm{GO}[44]$. Here, the side-pinning effect refers to the hydrogen bonds formed between water molecules and oxygen-containing groups on GO surface and acted as anchors preventing water permeation. We therefore count the number of hydrogen bonds formed between GO nanosheets and water in the nanochannels, as shown in Figure 6. In general, the number of hydrogen bonds is close with each other regardless of different interlayer spacing at a fixed OD, except the case of $\mathrm{d}=0.6 \mathrm{~nm}$. In this case, the number of hydrogen bonds reaches highly 97 , while the average value of $0.8 \sim 1.8 \mathrm{~nm}$ is 66 . Obviously, at a fixed interlayer spacing, the number of hydrogen bonds increase gradually with the increase of oxidation degree. For example, the numbers of hydrogen bonds are 0,46 , 67,76 and 88 , corresponding to the OD of $0,10 \%, 20 \%, 30 \%$ and $40 \%$ at a fixed $d=1.2 \mathrm{~nm}$ (Figure 6B).

Eventually, we depict the relationship between hydrogen bonds and pull forces. The results further confirm the side-pinning effect. The pull forces are directly proportional to the number of hydrogen bonds (Figure 7). In detail, the pull forces become weaker with the increasing interlayer spacing at a fixed OD. On the other hand, the pull forces get stronger with the increasing OD at a fixed interlayer spacing, since more and more hydrogen bonds are formed and prevent water molecules passing the channel. Hence, stronger forces are required to pull the plate at a stable speed. As a result, higher OD is not favorable to water transport through PG/GO nanochannels.

\section{Conclusions}

In summary, MD simulations have been conducted to investigate the effects of interlayer spacing and oxidation degree on water permeation through GO membranes. The MD results show that there is only one layer of water in the nanochannel when $d=0.6 \mathrm{~nm}$. When the interlayer spacing falls in the range of $0.8 \sim 1.0 \mathrm{~nm}$, two layers of water molecules formed between GO sheets. GO possesses strong adsorption capacity that these two layers of water are hard to flow on GO surface. When the interlayer spacing continues to increase, the structure of water molecules in the middle of nanochannel becomes disordered. It is clear that increasing interlayer spacing is beneficial to water flow, but is detrimental to ion sieving or molecular separation. We therefore propose that the most suitable interlayer spacing for water flow is $1.2 \mathrm{~nm}$, as there are just three layers of water formed between two GO sheets, and the middle layer of water molecules is relatively easy to flow. On the other hand, with the increasing oxidation degree, more and more hydrogen bonds are formed and prevent water flowing on GO surface. That is, oxygencontaining groups are unfavorable for water permeation. Further study should focus on the separation performance of GO membranes taking interlayer spacing and oxidation degree into consideration.

\section{Declarations}


Funding: This work was supported by the National Natural Science Foundation of China (12074341, U1832150, 11875236), the Fundamental Research Funds for the Provincial Universities of Zhejiang (2020TD001), and the Scientific Research and Developed Fund of Zhejiang A\&F University (No. 2017FR032).

Conflicts of Interest/competing interests: The authors declare no competing interests.

Availability of data and material: All data relevant to this work are deposited at the Dryad Data Repository: https://datadryad.org/stash/share/LIXuH17HtVEgmW2If2mpGJZ2xNt5loRdSM9iVH2fsZo

Code availability: GROMACS is free of charge and open source. http://www.gromacs.org/

Author's Contributions: Q.T. and Y.F. performed the simulations and wrote the paper. Z.S. analyzed the results and prepared all figures. J.C. and L. C. reviewed and revised the manuscript. All authors gave final approval for publication.

\section{References}

1. Shen J, Hu Y, Shi M et al (2009) Fast and Facile Preparation of Graphene Oxide and Reduced Graphene Oxide Nanoplatelets. Chem Mater 21:3514-3520. https://doi.org/10.1021/cm901247t

2. Cohen-Tanugi D, Grossman JC (2012) Water Desalination across Nanoporous Graphene. Nano Lett 12:3602-3608. https://doi.org/10.1021/nl3012853

3. Zhang H, Lv X, Li Y et al (2010) P25-Graphene Composite as a High Performance Photocatalyst. ACS Nano 4:380-386. https://doi.org/10.1021/nn901221k

4. Lee K, Lee H, Shin Y et al (2016) Highly transparent and flexible supercapacitors using graphenegraphene quantum dots chelate. Nano Energy 26:746-754.

https://doi.org/10.1016/j.nanoen.2016.06.030

5. Cohen-Tanugi D, Lin L-C, Grossman JC (2016) Multilayer Nanoporous Graphene Membranes for Water Desalination. Nano Lett 16:1027-1033. https://doi.org/10.1021/acs.nanolett.5b04089

6. Chen H, Müller MB, Gilmore KJ et al (2008) Mechanically Strong, Electrically Conductive, and Biocompatible Graphene Paper. Adv Mater 20:3557-3561.

https://doi.org/10.1002/adma.200800757

7. Zhao M, Yang X (2015) Segregation Structures and Miscellaneous Diffusions for Ethanol/Water Mixtures in Graphene-Based Nanoscale Pores. J Phys Chem C 119:21664-21673. https://doi.org/10.1021/acs.jpcc.5b03307

8. Surwade SP, Smirnov SN, Vlassiouk IV et al (2015) Water desalination using nanoporous single-layer graphene. Nat Nanotechnol 10:459-464. https://doi.org/10.1038/nnano.2015.37

9. Konatham D, Yu J, Ho TA, Striolo A (2013) Simulation Insights for Graphene-Based Water Desalination Membranes. Langmuir 29:11884-11897. https://doi.org/10.1021/la4018695 
10. Han Y, Xu Z, Gao C (2013) Ultrathin Graphene Nanofiltration Membrane for Water Purification. Adv Func Mater 23:3693-3700. https://doi.org/10.1002/adfm.201202601

11. Abdelkader AM, Kinloch IA, Dryfe R, a. W (2014) High-yield electro-oxidative preparation of graphene oxide. Chem Commun 50:8402-8404. https://doi.org/10.1039/C4CC03260H

12. HUMMERS WS (1958) Preparation of graphitic oxide. J Am Chem Soc 80:1339-1339. https://doi.org/10.1021/ja01539a017

13. Zhang L, Dai F, Yi R et al (2020) Effect of physical and chemical structures of graphene oxide on water permeation in graphene oxide membranes. Appl Surf Sci 520:146308. https://doi.org/10.1016/j.apsusc.2020.146308

14. Szabó T, Berkesi O, Forgó P et al (2006) Evolution of Surface Functional Groups in a Series of Progressively Oxidized Graphite Oxides. Chem Mater 18:2740-2749. https://doi.org/10.1021/cm060258+

15. Chen L, Shi G, Shen J et al (2017) lon sieving in graphene oxide membranes via cationic control of interlayer spacing. Nature 550:380-383. https://doi.org/10.1038/nature24044

16. Nair RR, Wu HA, Jayaram PN et al (2012) Unimpeded Permeation of Water Through Helium-LeakTight Graphene-Based Membranes. Science 335:442-444. https://doi.org/10.1126/science.1211694

17. Joshi RK, Carbone P, Wang FC et al (2014) Precise and Ultrafast Molecular Sieving Through Graphene Oxide Membranes. Science 343:752-754. https://doi.org/10.1126/science.1245711

18. Sun P, Zheng F, Zhu M et al (2014) Selective Trans-Membrane Transport of Alkali and Alkaline Earth Cations through Graphene Oxide Membranes Based on Cation $-\pi$ Interactions. ACS Nano 8:850859. https://doi.org/10.1021/nn4055682

19. Sun P, Zhu M, Wang K et al (2013) Selective Ion Penetration of Graphene Oxide Membranes. ACS Nano 7:428-437. https://doi.org/10.1021/nn304471w

20. Hu M, Mi B (2013) Enabling Graphene Oxide Nanosheets as Water Separation Membranes. Environ Sci Technol 47:3715-3723. https://doi.org/10.1021/es400571g

21. Dai F, Yu R, Yi R et al (2020) Ultrahigh water permeance of a reduced graphene oxide nanofiltration membrane for multivalent metal ion rejection. Chem Commun 56:15068-15071. https://doi.org/10.1039/D0CC06302A

22. Xu L, Hu Y, Ma T-B, Wang H (2013) Tunable giant anisotropic diffusion of water sub-monolayers between graphene layers. Nanotechnology 24:505504. https://doi.org/10.1088/0957$4484 / 24 / 50 / 505504$

23. Kolesnikov Al, Zanotti J-M, Loong C-K et al (2004) Anomalously soft dynamics of water in a nanotube: a revelation of nanoscale confinement. Phys Rev Lett 93:035503. https://doi.org/10.1103/PhysRevLett.93.035503

24. Wang R, Chen J, Chen $L$ et al (2020) Ultrathin and ultradense aligned carbon nanotube membranes for water purification with enhanced rejection performance. Desalination 494:114671. https://doi.org/10.1016/j.desal.2020.114671 
25. Chen J, Dai F, Zhang L et al (2020) Molecular insights into the dispersion stability of graphene oxide in mixed solvents: Theoretical simulations and experimental verification. J Colloid Interface Sci 571:109-117. https://doi.org/10.1016/j.jcis.2020.03.036

26. Gogoi A, Reddy KA, Mondal PK (2020) Influence of the presence of cations on the water and salt dynamics inside layered graphene oxide (GO) membranes. Nanoscale 12:7273-7283. https://doi.org/10.1039/C9NR09288A

27. Giri AK, Teixeira F, Cordeiro MNDS (2018) Structure and kinetics of water in highly confined conditions: A molecular dynamics simulation study. J Mol Liq 268:625-636. https://doi.org/10.1016/j.molliq.2018.07.083

28. Wang Y, He Z, Gupta KM et al (2017) Molecular dynamics study on water desalination through functionalized nanoporous graphene. Carbon 116:120-127. https://doi.org/10.1016/j.carbon.2017.01.099

29. Xiong W, Liu JZ, Ma M et al (2011) Strain engineering water transport in graphene nanochannels. Phys Rev E 84:056329. https://doi.org/10.1103/PhysRevE.84.056329

30. Qiao Y, Xu X, Li H (2013) Conduction of water molecules through graphene bilayer. Appl Phys Lett 103:233106. https://doi.org/10.1063/1.4839255

31. Boukhvalov DW, Katsnelson MI, Son Y-W (2013) Origin of Anomalous Water Permeation through Graphene Oxide Membrane. Nano Lett 13:3930-3935. https://doi.org/10.1021/nl4020292

32. Yi R, Xia X, Yang R et al (2021) Selective reduction of epoxy groups in graphene oxide membrane for ultrahigh water permeation. Carbon 172:228-235. https://doi.org/10.1016/j.carbon.2020.09.076

33. Dai H, Xu Z, Yang X (2016) Water Permeation and Ion Rejection in Layer-by-Layer Stacked Graphene Oxide Nanochannels: A Molecular Dynamics Simulation. J Phys Chem C 120:22585-22596. https://doi.org/10.1021/acs.jpcc.6b05337

34. Falk K, Sedlmeier F, Joly L et al (2010) Molecular Origin of Fast Water Transport in Carbon Nanotube Membranes: Superlubricity versus Curvature Dependent Friction. Nano Lett 10:4067-4073. https://doi.org/10.1021/nl1021046

35. Berendsen HJC, van der Spoel D, van Drunen R (1995) GROMACS: A message-passing parallel molecular dynamics implementation. Comput Phys Commun 91:43-56. https://doi.org/10.1016/0010-4655(95)00042-E

36. Abraham MJ, Murtola T, Schulz R et al (2015) GROMACS: High performance molecular simulations through multi-level parallelism from laptops to supercomputers. SoftwareX 1-2:19-25. https://doi.org/10.1016/j.softx.2015.06.001

37. Jorgensen WL, Maxwell DS, Tirado-Rives J (1996) Development and Testing of the OPLS All-Atom Force Field on Conformational Energetics and Properties of Organic Liquids. J Am Chem Soc 118:11225-11236. https://doi.org/10.1021/ja9621760

38. Safaei S, Tavakoli R (2017) On the design of graphene oxide nanosheets membranes for water desalination. Desalination 422:83-90. https://doi.org/10.1016/j.desal.2017.08.013 
39. Jorgensen WL, Chandrasekhar J, Madura JD et al (1983) Comparison of simple potential functions for simulating liquid water. J Chem Phys 79:926-935. https://doi.org/10.1063/1.445869

40. Essmann U, Perera L, Berkowitz ML et al (1995) A smooth particle mesh Ewald method. J Chem Phys 103:8577-8593. https://doi.org/10.1063/1.470117

41. Darden T, York D, Pedersen L (1993) Particle mesh Ewald: An $N \cdot \log (N)$ method for Ewald sums in large systems. J Chem Phys 98:10089-10092. https://doi.org/10.1063/1.464397

42. Hess B, Bekker H, Berendsen HJC, Fraaije JGEM (1997) LINCS: A linear constraint solver for molecular simulations. Journal of Computational Chemistry 18:1463-1472. https://doi.org/10.1002/(SICI)1096-987X(199709)18:12<1463::AID-JCC4>3.0.CO;2-H

43. Miyamoto S, Kollman PA (1992) Settle: An analytical version of the SHAKE and RATTLE algorithm for rigid water models. J Comput Chem 13:952-962. https://doi.org/10.1002/jcc.540130805

44. Wei N, Peng X, Xu Z (2014) Understanding Water Permeation in Graphene Oxide Membranes. ACS Appl Mater Interfaces 6:5877-5883. https://doi.org/10.1021/am500777b

\section{Figures}
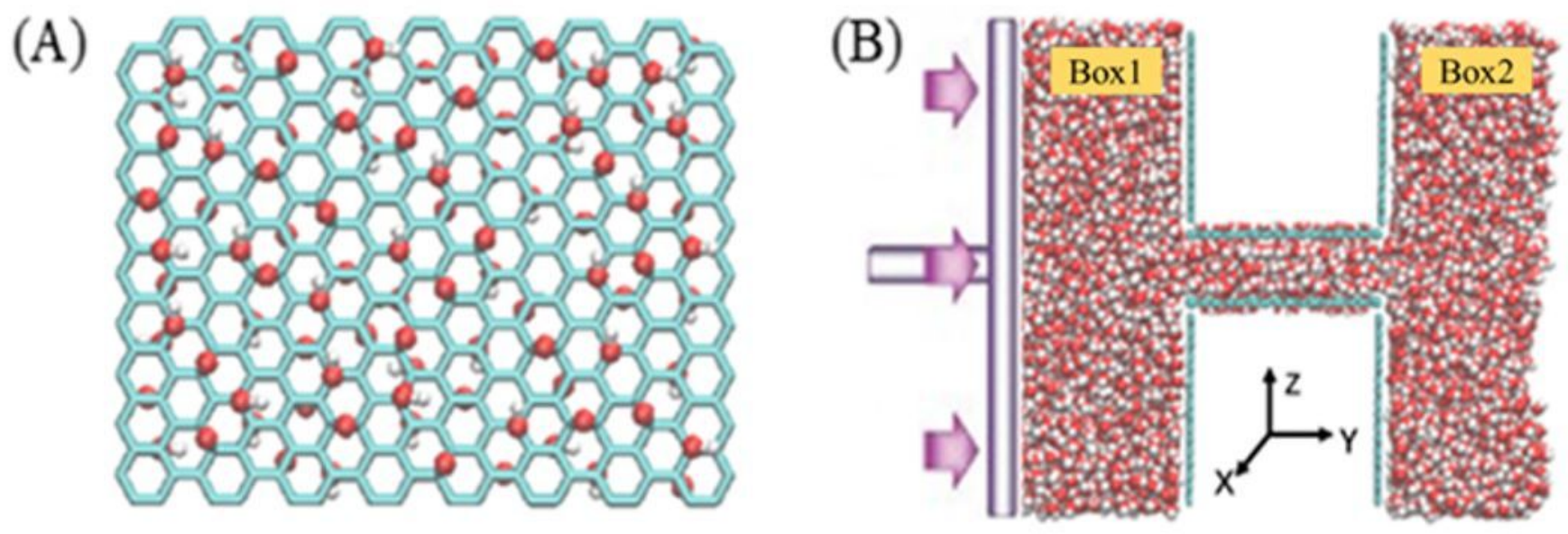

\section{Figure 1}

(A) Atomic structure of GO nanosheet with hydroxyl and epoxy groups. The red and white balls represent the oxygen and hydrogen atoms. (B) Side view of the simulation system composed of two GO nanosheets and two reservoirs. 

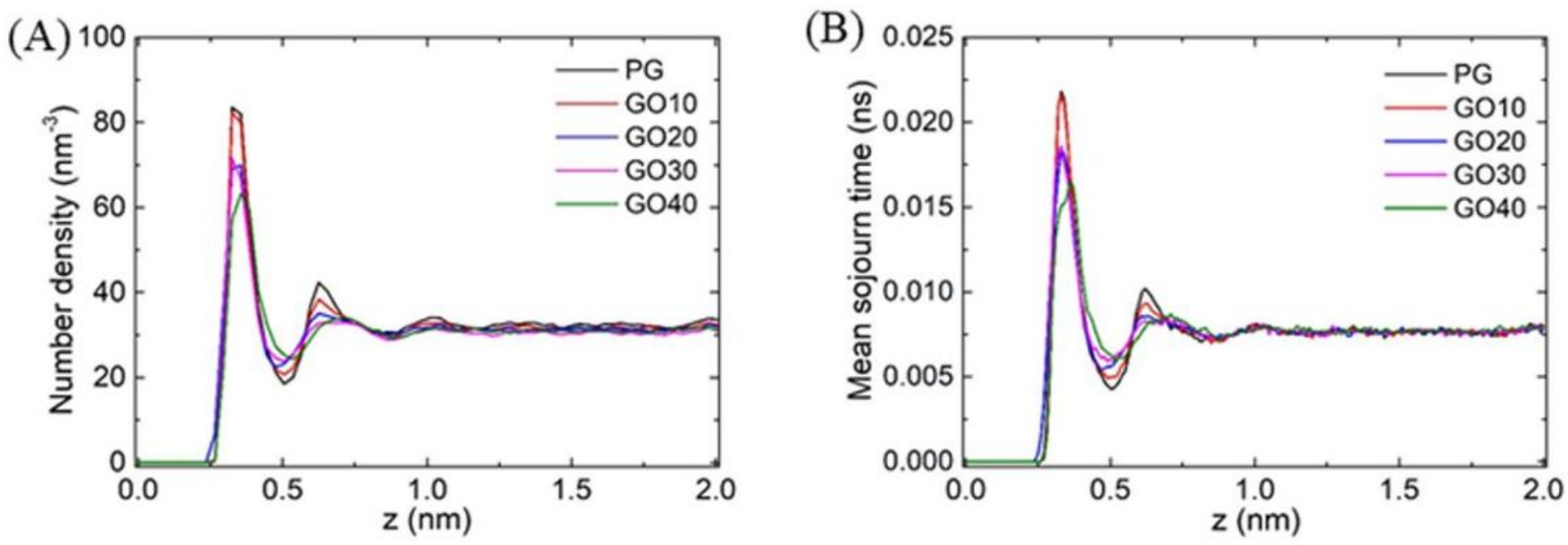

Figure 2

(A) Molecular number density profiles of water molecules in z direction (normal to PG/GO surface). (B) Mean sojourn time for water molecules with respect to their initial z coordinates.

(A)

(B)

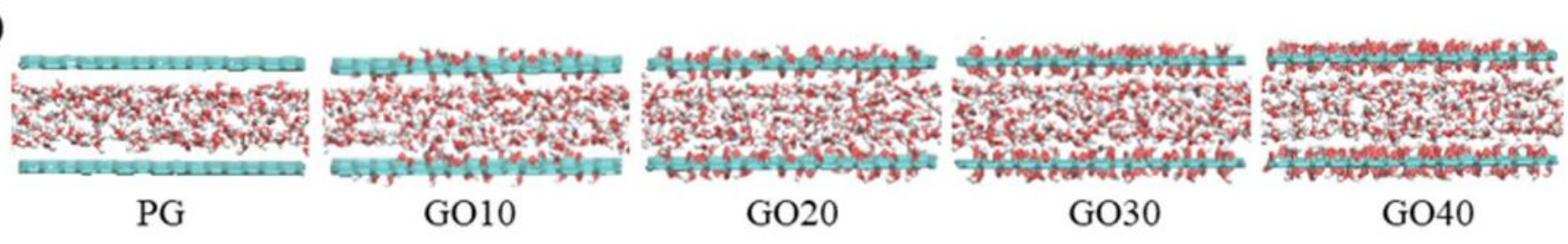

(C)

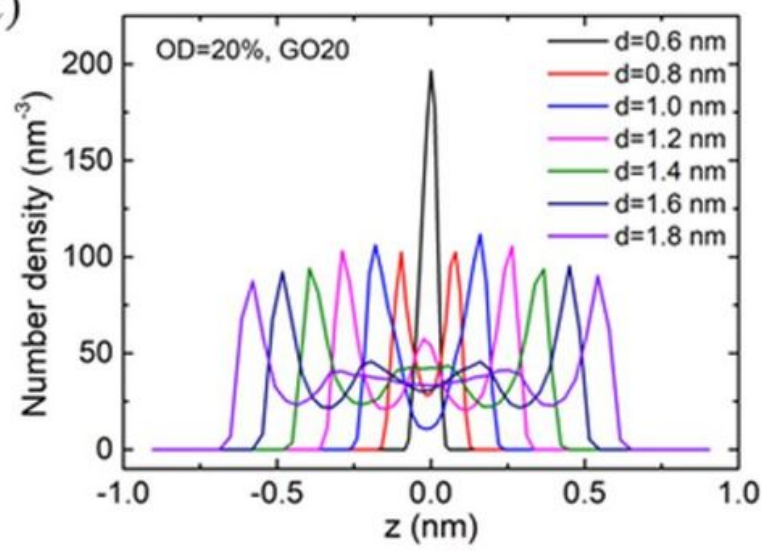

(D)

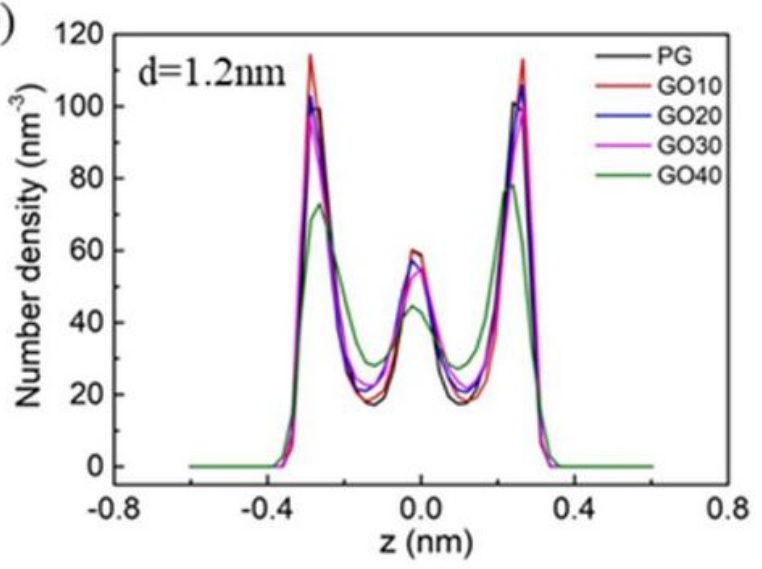

Figure 3

(A) Atomic structures of water molecules between two G020 sheets with varied interlayer spacing from $0.6 \mathrm{~nm}$ to $1.6 \mathrm{~nm}$. (B) Atomic structures of water molecules between PG/GO sheets with varied OD from 
$0 \%$ to $40 \%$. (C, D) The number density profiles along the thickness direction $z$, corresponding to panel $(A)$ and (B), respectively.
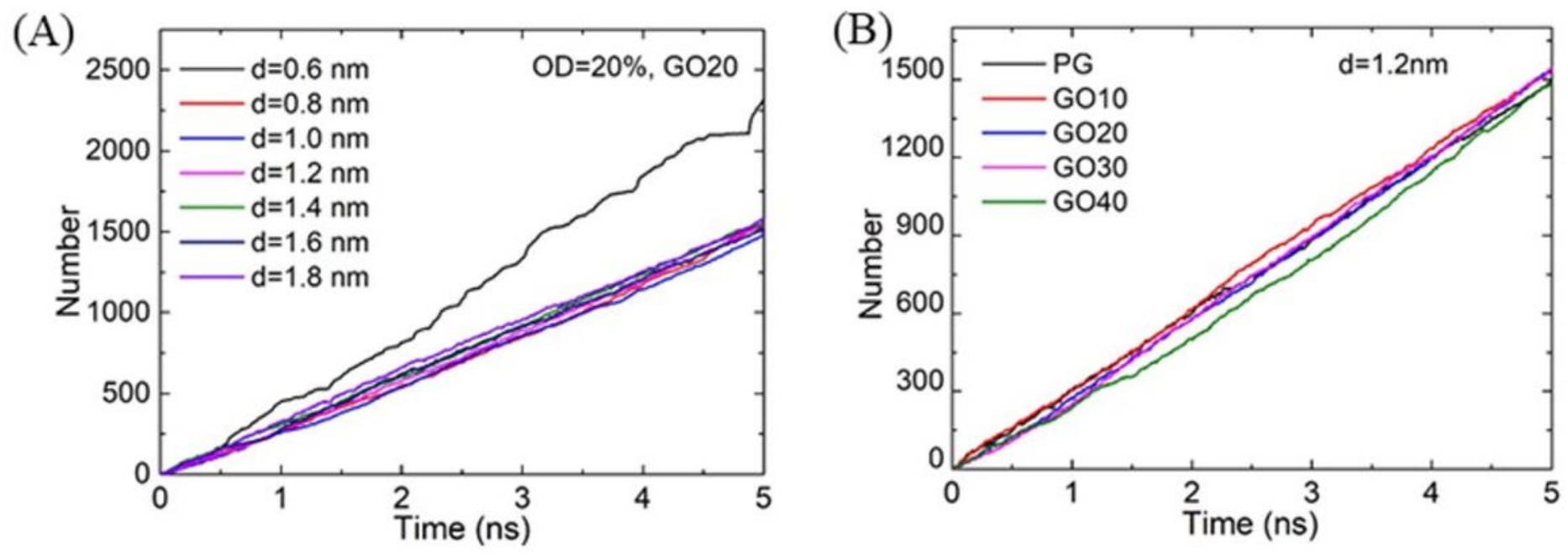

\section{Figure 4}

Water flow versus time for $(A)$ different width at $O D=20 \%$ and $(B)$ different $O D$ at $d=1.2 \mathrm{~nm}$.
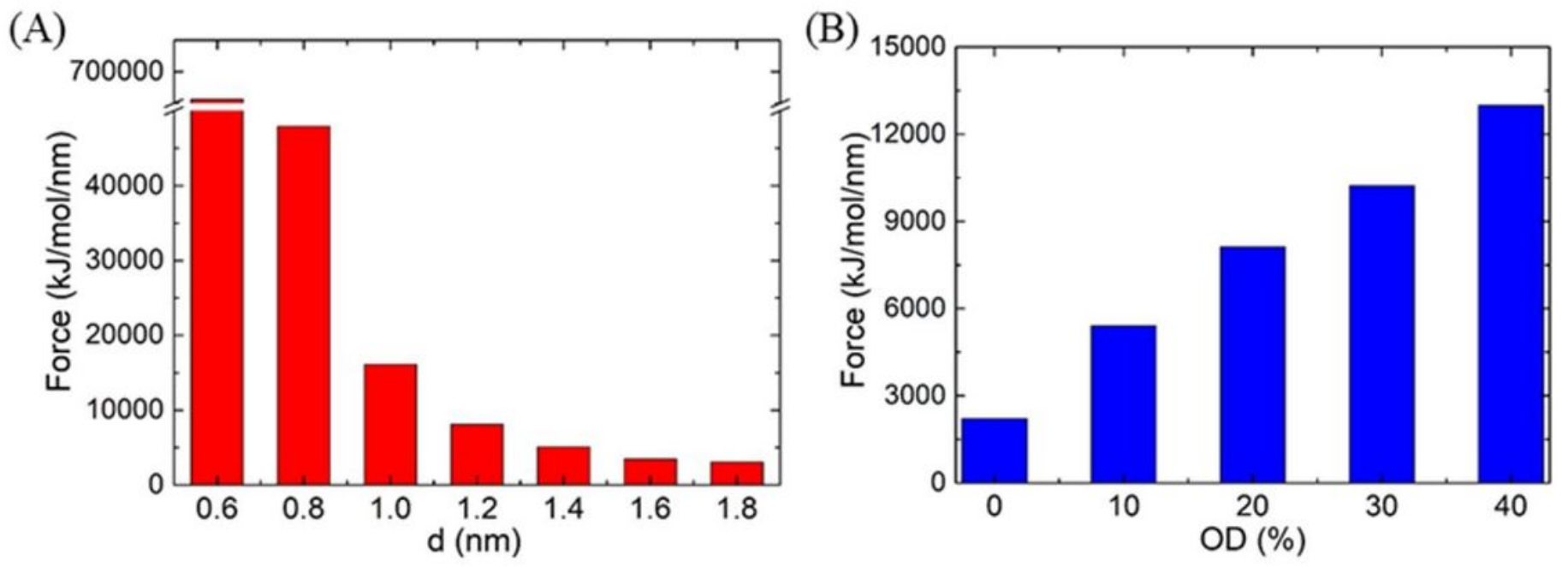

Figure 5

Cumulative average pull forces over the last $5 \mathrm{~ns}$ for $(\mathrm{A})$ different width at $\mathrm{OD}=20 \%$ and (B) different $\mathrm{OD}$ at $\mathrm{d}=1.2 \mathrm{~nm}$. 

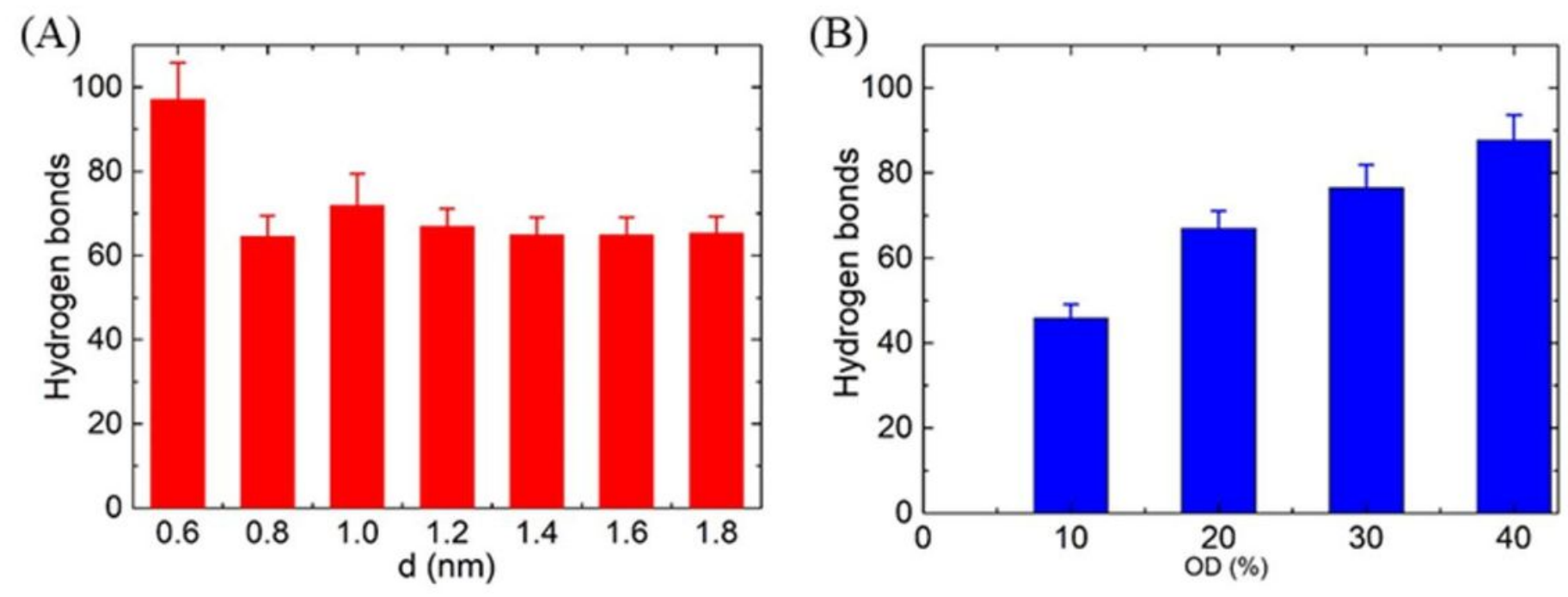

Figure 6

The averaged number of hydrogen bonds over the last $5 \mathrm{~ns}$ for $(A)$ different width at $O D=20 \%$ and $(B)$ different $O D$ at $d=1.2 \mathrm{~nm}$.
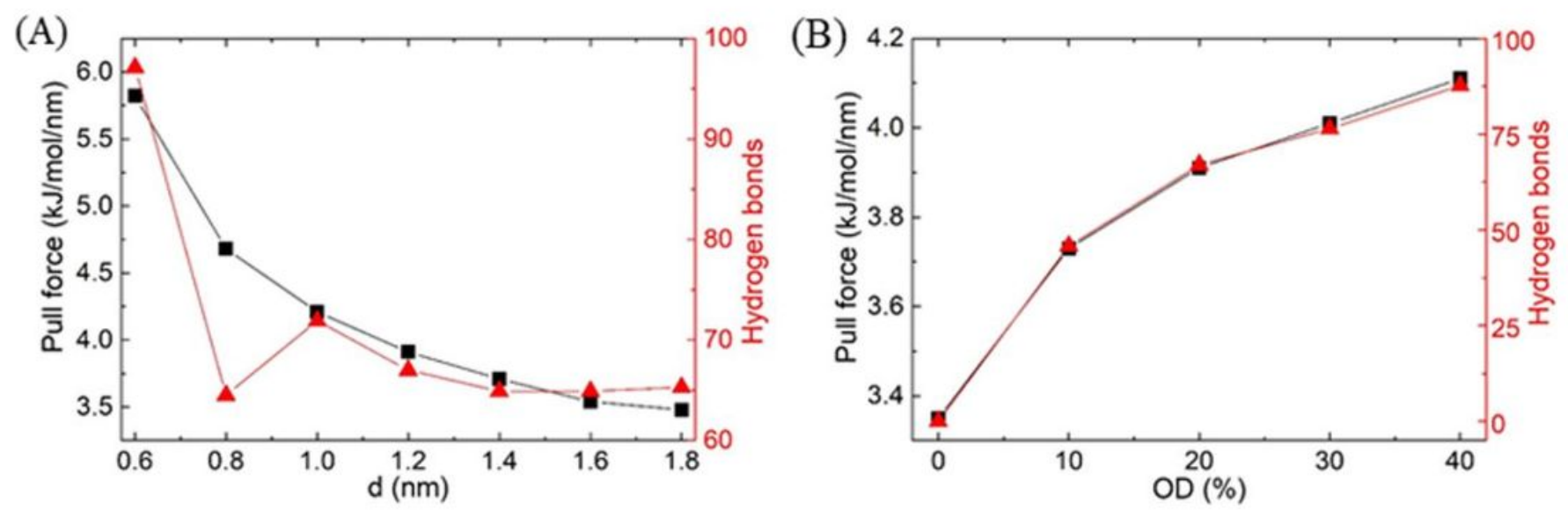

Figure 7

The relationship between hydrogen bonds and logarithm pull forces (A) different width at OD $=20 \%$ and (B) different OD at $\mathrm{d}=1.2 \mathrm{~nm}$. 